\title{
O papel da atenção primária na rede de atenção à saúde no Brasil: limites e possibilidades no enfrentamento da COVID-19
}

\section{The role of primary care in the Brazilian healthcare system: limits and possibilities for fighting COVID-19}

\author{
El papel de la atención primaria en el sistema de \\ salud brasileño: límites y posibilidades para \\ combatir COVID-19
}

\author{
Regina Paiva Daumas 1 \\ Gulnar Azevedo e Silva 2 \\ Renato Tasca 3 \\ Iuri da Costa Leite 1 \\ Patrícia Brasil 4 \\ Dirceu B. Greco 5 \\ Victor Grabois 1 \\ Gastão Wagner de Sousa Campos 6
}

doi: 10.1590/0102-311X00104120

\section{Introdução}

Em 12 de março de 2020, a Organização Mundial da Saúde (OMS) declarou como pandemia a doença causada pelo novo coronavírus (SARS-CoV-2) 1. A infecção (COVID-19), além de ser mais transmissível, tem letalidade estimada em cerca de 14 vezes a da influenza 2 . O alto índice de contágio, com cada indivíduo infectando de 2 a 3 pessoas, em média, causa a expansão da epidemia em progressão geométrica ${ }^{3}$. No Brasil, onde a transmissão comunitária em todo o território nacional foi declarada em 20 de março, mais de 147 mil casos e 10 mil mortes pela COVID-19 haviam sido confirmados até 9 de maio de 2020 4. Medidas de isolamento de casos e contatos e distanciamento social da população geral têm sido as principais estratégias preconizadas para retardar a expansão da COVID-19 e permitir a adequação dos sistemas de saúde ao rápido aumento da demanda por leitos de internação, especialmente aqueles de terapia intensiva, evitando o colapso da assistência hospitalar 5,6.

A apresentação inicial da COVID-19 se assemelha a uma gripe, com sintomas de febre, tosse, dor de garganta e coriza. Aproximadamente $80 \%$ dos pacientes se recuperam sem complicações, sendo classificados como casos leves ou moderados (sem pneumonia ou com pneumonia viral leve) ${ }^{7}$. Os $20 \%$ restantes evoluem com dispneia e hipoxemia secundárias à pneumonia viral extensa, e necessitam de internação para oxigenioterapia, além de outras intervenções ${ }^{8}$. Um quarto desses (cerca de $5 \%$ do total) evolui para o estado crítico devido à insuficiência respiratória, coagulação intravascular disseminada, choque circulatório ou disfunção orgânica múltipla, requerendo cuidados de terapia intensiva. A letalidade nesse último grupo é superior a 40\% 9,10.

A assistência à COVID-19 precisa dar conta das necessidades dos pacientes nas diferentes fases da infecção e em todo o espectro de gravidade, em uma linha de cuidado que envolve desde o monitoramento de casos leves em isolamento domiciliar, com orientações para o manejo de sintomas e para a identificação precoce de sinais de alerta, até a internação em unidades de terapia intensiva (UTI) e a reabilitação após a alta hospitalar. A minimização dos riscos de infecção dos profissionais de saúde e demais pacientes deve balizar a escolha das melhores estratégias de organização da rede para atender a essas necessidades 11 .

Para reduzir a transmissão e limitar a mortalidade pela COVID-19, o poder público deve criar condições para que indivíduos e famílias possam sustentar o distanciamento social enquanto prepara
1 Escola Nacional de Saúde Pública Sergio Arouca, Fundação Oswaldo Cruz, Rio de Janeiro, Brasil. 2 Instituto de Medicina Social, Universidade do Estado do Rio de Janeiro, Rio de Janeiro, Brasil. 3 Organização Pan-Americana da Saúdel Organização Mundial da Saúde, Brasília, Brasil. 4 Instituto Nacional de Infectologia Evandro Chagas, Fundação Oswaldo Cruz, Rio de Janeiro, Brasil.

5 Faculdade de Medicina, Universidade Federal de Minas Gerais, Belo Horizonte, Brasil.

${ }^{6}$ Faculdade de Ciências Médicas, Universidade Estadual de Campinas, Campinas, Brasil.

Correspondência I. C. Leite

Escola Nacional de Saúde Pública Sergio Arouca, Fundação Oswaldo Cruz. Rua Leopoldo Bulhões 1480, Rio de Janeiro, RJ 21041-210 Brasil.

iuri.fiocruz@gmail.com 
seu sistema de saúde para o enfrentamento da pandemia. No caso do Brasil, o Sistema Único de Saúde (SUS) deve se preparar para prover assistência ao crescente número de pacientes com a COVID-19; manter a atenção aos demais agravos agudos e crônicos e garantir a segurança de profissionais de saúde e pacientes durante o cuidado de saúde 12 .

\section{Recomendações para a organização da rede de atenção à COVID-19}

A capacidade do sistema de saúde de desempenhar plenamente suas funções no contexto da pandemia demanda não apenas expandir o número de leitos hospitalares e de UTI, mas também reorganizar os fluxos na rede de atendimento, redefinir os papéis das diferentes unidades e níveis de atenção e criar novos pontos de acesso ao sistema de saúde, especialmente por via remota 11 . Todas as modalidades de teleatendimento (teleorientação, teleconsulta, telemonitoramento e telerregulação) passam a desempenhar papel central a partir desse momento 13,14. Um plano com protocolos a serem seguidos por todos os níveis de atenção, incluindo normas de proteção aos trabalhadores e cuidados para evitar a disseminação do SARS-CoV-2 nas unidades de saúde, é fundamental para articular todas essas ações 15.

A OMS sugere que sejam criadas centrais de teleatendimento 24 horas, treinando os atendentes na utilização de protocolos que permitam distinguir casos leves, moderados e graves, bem como orientar os casos leves quanto às medidas de isolamento domiciliar. Casos graves devem ser encaminhados para hospitais de referência para COVID-19 por ambulâncias exclusivas para este transporte, com profissionais qualificados e adequadamente protegidos, segundo as medidas preconizadas para a prevenção de infecção 11 .

Na rede de atenção à COVID-19, são também necessários equipamentos de saúde com leitos de baixa e média complexidades dedicados ao atendimento de casos suspeitos com alto risco de agravamento ou com contraindicação ao isolamento domiciliar, tais como aqueles com comorbidades, os que residem sozinhos e os que apresentam maior comprometimento pela doença, ainda que não estejam graves. A internação nesses locais possibilita fazer intervenções terapêuticas, monitorar sinais de agravamento e providenciar transferência oportuna para leitos de maior complexidade, quando necessário. Essas unidades devem admitir também pacientes que recebam alta de hospitais de referência ainda necessitando de cuidados hospitalares, contribuindo para a liberação dos leitos de alta complexidade para os pacientes críticos 11,16 .

A descrição das necessidades assistenciais dos pacientes com COVID-19 torna evidente que o papel direto da atenção primária à saúde (APS) na assistência aos casos moderados e graves é bastante limitado. Ademais, o atendimento presencial aos pacientes suspeitos de COVID-19 nas unidades básicas de saúde (UBS) deve ser evitado sempre que possível porque, além de ter pouco impacto no curso da doença, implica alto risco de infecção para os profissionais de saúde e demais usuários.

\section{O papel da APS durante a pandemia de COVID-19}

Se por um lado a APS tem capacidade reduzida para atuar sobre a letalidade dos casos graves, uma APS forte, organizada e com pessoal qualificado e em número adequado pode contribuir para diminuir a incidência da infecção na população adscrita, com impacto direto na diminuição da morbimortalidade. Por meio do trabalho comunitário pode atuar para a redução da disseminação da infecção, acompanhar os casos leves em isolamento domiciliar, apoiar as comunidades durante o distanciamento social, identificar e conduzir situações de vulnerabilidade individual ou coletiva e, principalmente, garantir o acesso a cuidados de saúde e o necessário encaminhamento nas fases mais críticas da epidemia. Dessa forma, a APS pode desempenhar um papel central na mitigação dos efeitos da pandemia, mantendo e aprofundando todos os seus atributos, tais como o acesso ao primeiro contato, a longitudinalidade, a integralidade e a coordenação do cuidado e, em especial, a competência cultural e a orientação familiar e comunitária ${ }^{17}$.

Ao retomar a sua vocação para a ação comunitária, a APS pode ampliar a capacidade de resposta local não só para reduzir a disseminação da infecção, como também para amenizar os efeitos sociais e econômicos das medidas de distanciamento social. Por exemplo, por intermédio das mídias sociais 
e rádios comunitárias, as equipes de saúde da família $(\mathrm{EqSF})$ podem orientar a população sobre as formas de contágio e informar sobre os canais de teleatendimento. $\mathrm{O}$ trabalho dos agentes comunitários de saúde (ACS) pode ajudar a identificar indivíduos e famílias em situação de maior vulnerabilidade, auxiliar na entrega de alimentos e outros itens essenciais, além de mobilizar os recursos da própria comunidade.

$\mathrm{Na}$ atenção clínica individual, utilizando modalidades de teleatendimento, profissionais da APS podem orientar os casos suspeitos quanto ao isolamento e reconhecimento dos sinais de alerta; identificar pacientes que não podem ser cuidados no domicílio; monitorar estes casos suspeitos quanto à evolução clínica; realizar videoconsultas para casos mais complexos e solicitar remoção para uma unidade hospitalar ao identificar sinais de agravamento. Todas essas ações resultam em redução da demanda e dos riscos de infecção nas unidades de emergência e permitem a concentração de seus recursos no atendimento aos casos mais graves.

Visando a manter o acesso aos cuidados de saúde para outros agravos, o trabalho na APS durante a pandemia deve priorizar ainda: (1) a continuidade de ações preventivas, tais como vacinação; (2) o acompanhamento de pacientes crônicos e grupos prioritários como gestantes e lactentes; e (3) o atendimento a pequenas urgências e às agudizações de doenças crônicas. A supressão dessas atividades por várias semanas pode resultar em elevação da morbimortalidade por outras causas, ampliando os efeitos da pandemia, como relatado nas situações em que se optou por um modelo assistencial centrado apenas na atenção hospitalar 18.

Assim como para os casos suspeitos, o teleatendimento deve ser priorizado como estratégia para o acompanhamento dos portadores de outros agravos que estão estáveis. É possível responder a demandas por medicamentos de uso contínuo ou mesmo por avaliação clínica, que pode, muitas vezes, ser realizada por videoconsulta. $\mathrm{O}$ atendimento presencial na UBS seria priorizado para pacientes com queixas agudas não respiratórias e para aqueles com agudização de doenças crônicas, cuja abordagem possa ser feita na APS, com até algumas horas de tratamento e observação clínica, evitando sua ida para unidades hospitalares e de pronto-atendimento, que estarão progressivamente sobrecarregadas. A disponibilização de equipamentos de proteção individual e a atualização das práticas de prevenção e controle de infecção de acordo com as melhores evidências disponíveis, com treinamentos periódicos, dão suporte à manutenção segura das ações assistenciais na UBS e nos domicílios.

Além do teleatendimento, a consulta domiciliar por médicos e enfermeiros pode garantir a manutenção da assistência a pacientes de maior complexidade e risco, incluindo ainda aqueles que necessitam de curativos. As visitas domiciliares feitas pelos ACS, no peridomicílio, respeitando o distanciamento social, possibilitam o acompanhamento de pacientes sem telefone e a entrega de medicamentos e de insumos básicos à população, evitando, assim, as visitas desnecessárias à UBS.

Mesmo com todo o esforço para expandir o uso das teleconsultas, e ainda que centrais telefônicas para o atendimento a casos suspeitos de COVID-19 sejam priorizadas, uma parcela substancial da população do território continuará acessando as UBS, Unidades de Pronto Atendimento (UPAs) e emergências hospitalares. Por esse motivo, os pacientes devem ser rastreados no primeiro ponto de contato com qualquer serviço de saúde e todas as precauções para o controle de infecção devem ser ali implementadas, de acordo com as recomendações vigentes, que incluem atendimento em áreas externas, limitação do contato físico, modificações de fluxo, separação de áreas de atendimento e espera, distanciamento, barreiras físicas, disponibilização e uso racional de equipamentos de proteção individual (EPI), de acordo com a atividade e tipo de contato realizado 1. Cabe lembrar ainda que, à medida que a epidemia avança, o risco de infecção em unidades de saúde aumenta, mesmo quando se trata de pacientes sem relato de sintomas respiratórios, pois casos de infecção assintomática e apresentações atípicas passam a ser mais numerosos. Isso justifica a expansão do uso de EPI e de medidas de controle de infecção para diversas situações e as áreas de atendimento 19.

Os riscos da transmissão da COVID-19 nos serviços de saúde e os elevados custos da implantação das normas de biossegurança justificam a ampla utilização das ferramentas de teleatendimento e todo o suporte financeiro necessário para implantá-las. Essas estratégias permitem que a restrição e regulação do acesso físico às UBS não representem restrição total de acesso, mas possam ser substituídas por formas mais seguras e custo-efetivas de atenção. Para implantar as mudanças necessárias ao pleno funcionamento da APS no contexto da pandemia, investimentos em estruturas como tendas 
para atendimento externo, veículos para apoiar a atenção domiciliar, telefones celulares e internet de boa qualidade serão imprescindíveis, assim como o acesso livre à internet para todos os usuários.

A criação de polos de atenção à COVID-19, destinados a atender os casos suspeitos, realizar o manejo inicial e facilitar as remoções necessárias, é uma alternativa válida onde seja impossível adequar as UBS para realizar essas atividades de forma segura 11. Não é possível dispensar, entretanto, a troca efetiva de informações entre esses polos e as UBS, essencial para que estas possam manter a vigilância e a coordenação do cuidado em suas respectivas áreas de abrangência. Somente uma APS forte e conectada aos demais pontos de atenção, com maior atuação comunitária e capacidade de coordenação, será capaz de minimizar os danos causados pela pandemia e evitar a desassistência às condições crônicas e agudizações.

\section{Limites da atuação da APS diante das deficiências da rede de atenção a COVID-19}

O pleno exercício de atividades inerentes à APS que poderiam reduzir a magnitude e o impacto da pandemia nas populações é ameaçado, contudo, pela deficiência da rede de atenção à COVID-19, notadamente pela insuficiência de leitos para internação e por prolemas no fluxo de acesso a esses leitos.

Por exemplo, no Município do Rio de Janeiro, onde o Serviço de Atendimento Móvel de Urgência (SAMU) não realiza o atendimento domiciliar às urgências por COVID-19 e as UBS são responsáveis por acolher os sintomáticos respiratórios e solicitar vagas de internação, casos de COVID-19, especialmente os casos mais graves se acumulam à espera de leitos de internação. Processo semelhante ocorre nas UPAs, outra porta de entrada física para os leitos Covid, que já enfrentam dificuldade crescente em referenciar seus pacientes para leitos especializados 20.

Ainda no mesmo município, a ausência de leitos básicos e intermediários para COVID-19 tem feito com que muitos pacientes regulados para as UPAs retornem às UBS, sempre em piores condições. A impossibilidade de internação de pacientes sem sinais de insuficiência respiratória, mas com alto risco de agravamento ou contraindicação ao isolamento domiciliar 21 , tem retardado a instituição de suporte clínico adequado e causado enorme apreensão nas equipes de saúde, que percebem o risco de pacientes chegarem às UBS em falência respiratória ou irem a óbito no domicílio sem assistência médica.

A insuficiência de leitos dedicados a COVID-19, aliada ao fluxo de acesso a estes leitos via UBS e UPAs, tal como ocorre no Município do Rio de Janeiro, tende a comprometer gravemente a capacidade destas unidades assistenciais de prover cuidados de saúde aos pacientes portadores de outras doenças e eleva os riscos de transmissão. O transporte dos pacientes graves do domicílio diretamente para a unidade de referência para tratamento de COVID-19, conforme sugere a OMS 11, deve ser considerado de extrema prioridade, pois agiliza a chegada ao local que poderá instituir a terapia definitiva e reduz os pontos de contato do paciente com as unidades de saúde e equipes de transporte, muito vulneráveis à infecção pelo SARS-CoV-2.

\section{Desafios para o SUS no enfrentamento à pandemia}

O enfrentamento da pandemia no país pressupõe mudanças substanciais na forma como os cuidados de saúde são prestados e a reorganização de toda a rede assistencial. Para otimizar o uso dos recursos disponíveis, as estruturas de teleatendimento precisam estar conectadas com o atendimento pré-hospitalar (SAMU-COVID) e a regulação de leitos pelo SUS. Protocolos de triagem e classificação dos casos em leves, moderados e graves, com ou sem risco de complicações, devem ser implantados para apoiar tanto as decisões do teleatendimento de emergência quanto a APS. Comunicações completas e consistentes entre todos os componentes do sistema de saúde e para a população são essenciais e devem ser urgentemente estabelecidas para garantir sua efetividade e transparência 13 .

Ainda que grandes mudanças nos fluxos e organização da rede de atenção possam parecer difíceis, todas as evidências indicam que fazer mais do mesmo significará maior disseminação da doença e ineficiência na utilização de recursos. A capacidade do sistema de saúde para salvar vidas nesta pandemia dependerá não apenas do número de leitos de UTI e respiradores, mas também da organização 
da rede de atenção para garantir o acesso oportuno a estes leitos, a manutenção das linhas de cuidado para outros agravos e o atendimento a urgências e emergências das mais diversas naturezas. As soluções específicas para essa organização devem ser adaptadas a cada contexto, respeitando premissas gerais e a capacidade de resposta local, entendendo que o Brasil é um país continental e heterogêneo. Para as grandes metrópoles, onde a doença tem avançado mais rápido, muito pode ser feito com as estruturas já existentes, complementadas por novos aportes e iniciativas.

É preciso reconhecer ainda que esse esforço de reorganização será limitado em seus resultados, caso não sejam resolvidas as deficiências de nossas redes de atenção, frágeis e fragmentadas, com uma APS ainda insuficiente - em muitos lugares do Brasil - para enfrentar uma emergência como esta, que depende de serviços efetivos e projetados à comunidade. Apesar da expansão da APS alcançada nas últimas décadas, a ausência de mecanismos para garantir sua sustentabilidade segue ameaçando a continuidade do cuidado em todo o país 22 . A falta de coordenação entre os níveis assistenciais e a inexistência de fluxos sistêmicos na rede de atenção à saúde, já apontadas anteriormente 23 , ficaram ainda mais críticas na pandemia, revelando a importância de se adotar estratégias que possibilitem às equipes de APS a efetiva coordenação do cuidado.

A epidemia chegou ao país após o inaceitável estabelecimento do teto de gastos para saúde e educação (Emenda Constitucional no 95 24), que congelou ou diminuiu os recursos para saúde. Ao mesmo tempo, políticas importantes e estruturantes para APS foram atacadas seriamente. Entre essas, destaca-se o Programa Mais Médicos pelo Brasil, criado para promover atendimento médico em lugares remotos e em periferias urbanas, cuja descontinuidade agravou os vazios assistenciais em locais altamente vulneráveis. Em sequência, o novo modelo de financiamento para a atenção básica 25 , imposto pelo atual Governo Federal, coloca em risco a universalidade do sistema 22. A despeito de que novos recursos possam ser alocados para o SUS durante a pandemia, não podemos ignorar o impacto deletério do crônico e histórico subfinanciamento do SUS sobre a saúde da população, que em sua maioria depende exclusivamente deste sistema.

Mesmo com suas deficiências, a importância do SUS no enfrentamento da pandemia tem sido demonstrada de forma inquestionável. O reconhecimento, agora quase unânime, da necessidade de mais recursos para que o sistema faça frente à crise torna este momento especialmente oportuno para reforçar e financiar adequadamente o SUS. Além disso, esta gravíssima emergência sanitária coloca em destaque a posição privilegiada da APS para garantir o acesso a cuidados de saúde e agir sobre os determinantes de saúde frente a um desafio emergente. A priorização da APS, com a expansão da Estratégia Saúde da Família e o fortalecimento de todos os seus atributos, com ênfase nos chamados derivados (competência cultural, orientação familiar e comunitária) 17, são medidas essenciais para conter a propagação na população de uma ameaça como a COVID-19.

\section{Colaboradores}

R. P. Daumas contribuiu com a concepção do estudo, elaboração e revisão do texto. G. Azevedo e Silva, R. Tasca, I. C. Leite, P. Brasil, D. B. Greco, V. Grabois e G. W. S. Campos contribuíram com a elaboração e revisão do texto.

\section{Informações adicionais}

ORCID: Regina Paiva Daumas (0000-0002-89237711); Gulnar Azevedo e Silva (0000-0001-87342799); Renato Tasca (0000-0002-1407-5914); Iuri da Costa Leite (0000-0002-9136-8948); Patrícia Brasil (0000-0001-9555-7976); Dirceu B. Greco (0000-0002-4419-5634); Victor Grabois (00000003-3310-4212); Gastão Wagner de Sousa Campos (0000-0001-5195-0215). 


\section{Referências}

1. World Health Organization. WHO announces COVID-19 outbreak a pandemic. http:// www.euro.who.int/en/health-topics/healthemergencies/coronavirus-covid-19/news/ news/2020/3/who-announces-covid-19-out break-a-pandemic (acessado em 18/Abr/2020).

2. Verity R, Okell LC, Dorigatti I, Winskill P, Whittaker C, Imai $\mathrm{N}$, et al. Estimates of the severity of coronavirus disease 2019: a model-based analysis. Lancet Infect Dis 2020; 20:P669-77.

3. Li Q, Guan X, Wu P, Wang X, Zhou L, Tong Y, et al. Early transmission dynamics in Wuhan, China, of novel coronavirus-infected pneumonia. N Engl J Med 2020; 382:1199-207.

4. Dong E, Du H, Gardner L. An interactive webbased dashboard to track COVID-19 in real time. Lancet Infect Dis 2020; 20:533-4.

5. Greenstone M, Nigam V. Does social distancing matter? https://papers.ssrn.com/ab stract $=3561244$ (acessado em 24/Abr/2020).

6. Prem K, Liu Y, Russell TW, Kucharski AJ, Eggo RM, Davies N, et al. The effect of control strategies to reduce social mixing on outcomes of the COVID-19 epidemic in Wuhan, China: a modelling study. Lancet Public Health 2020; 5:e261-e70.

7. Wu Z, McGoogan JM. Characteristics of and important lessons from the coronavirus disease 2019 (COVID-19) outbreak in China: summary of a report of 72314 cases from the Chinese Center for Disease Control and Prevention. JAMA 2020; 323:1239-42.

8. Negri EM, Piloto B, Morinaga LK, Jardim CVP, Lamy SAE-D, Ferreira MA, et al. Heparin therapy improving hypoxia in COVID-19 patients - a case series. medRxiv 2020; 22 abr. https:// www.medrxiv.org/content/10.1101/2020.04.1 $5.20067017 \mathrm{v} 3$.

9. Centers for Disease Control and Prevention. Interim clinical guidance for management of patients with confirmed coronavirus disease (COVID-19). https://www.cdc.gov/ coronavirus/2019-ncov/hcp/clinical-guid ance-management-patients.html (acessado em 24/Abr/2020).

10. World Health Organization. Clinical care for severe acute respiratory infection: toolkit: COVID-19 adaptation. Geneva: World Health Organization; 2020.

11. World Health Organization. Operational considerations for case management of COVID-19 in health facility and community: interim guidance. http://www.euro.who.int/en/health-top ics/health-emergencies/coronavirus-covid-19/ technical-guidance/2020/operational-con siderations-for-case-management-of-covid19-in-health-facility-and-community-interimguidance,-19-march-2020 (acessado em 11/ Mai/2020).
12. Cho SY, Kang J-M, Ha YE, Park GE, Lee JY, Ko J-H, et al. MERS-CoV outbreak following a single patient exposure in an emergency room in South Korea: an epidemiological outbreak study. Lancet 2016; 388:994-1001.

13. Centers for Disease Control and Prevention. Outpatient and ambulatory care settings: responding to community transmission of COVID-19 in the United States. https://www. cdc.gov/coronavirus/2019-ncov/hcp/ambula tory-care-settings.html (acessado em 24/Abr/ 2020).

14. Greenhalgh T, Koh GCH, Car J. Covid-19: avaliação remota em Atenção Primária à Saúde. Rev Bras Med Fam Comunidade 2020; 15:2461.

15. Associação Brasileira de Saúde Coletiva. Entidades enviam carta ao ministro sobre o risco de disseminação da Covid-19 nas unidades básicas de saúde. https://www.abrasco.org.br/site/no ticias/sistemas-de-saude/carta_ms_risco_ubs_ covid_19/46432/ (acessado em 28/Abr/2020).

16. Organización Panamericana de la Salud. Recomendaciones para la reorganización y ampliación progresiva de los servicios de salud para la respuesta a la pandemia de COVID-19. https:// www.paho.org/en/documents/recomendacio nes-para-reorganizacion-ampliacion-progresi va-servicios-salud-para-respuesta (acessado em 19/Abr/2020).

17. Starfield B. Atenção primária: equilíbrio entre necessidades de saúde, serviços e tecnologia. http://bases.bireme.br/cgi-bin/wxislind.exe/ iah/online/? IsisScript=iah/iah.xis\&src=google \&base $=$ LILACS\&lang $=$ p\&nextAction $=\operatorname{lnk} \&$ ex prSearch $=622019 \&$ indexSearch $=$ ID (acessado em 24/Abr/2020).

18. Nacoti M, Ciocca A, Giupponi A, Brambillasca P, Lussana F, Pisano M, et al. At the epicenter of the Covid-19 pandemic and humanitarian crises in Italy: changing perspectives on preparation and mitigation. https://catalyst.nejm.org/ doi/pdf/10.1056/CAT.20.0080 (acessado em 27/Mar/2020).

19. Public Health England. COVID-19 personal protective equipment (PPE). https://www.gov. uk/government/publications/wuhan-novelcoronavirus-infection-prevention-and-con trol/covid-19-personal-protective-equipmentppe (acessado em 19/Abr/2020).

20. Altino L. Com UPAs lotadas, pacientes com falta de oxigênio aguardam horas e enfrentam até tiroteio antes de transferência. Extra Online 2020; 22 abr. https://extra.globo.com/noticias/ coronavirus/com-upas-lotadas-pacientes-comfalta-de-oxigenio-aguardam-horas-enfrentamate-tiroteio-antes-de-transferencia-24388299. html. 
21. Prefeitura Municipal do Rio de Janeiro. Resolução SMS no 4.345 de 30 de março de 2020. Aprova e concede efeito normativo à Nota Técnica Conjunta SUBREG e SUBHUE - COVID -19 de 27 de março de 2020. Diário Oficial do Município do Rio de Janeiro 2020; 31 mar.

22. Giovanella L, Franco CM, Almeida PF. Política Nacional de Atenção Básica: para onde vamos? Ciênc Saúde Colet 2020; 25:1475-82.

23. Viana ALd'A, Bousquat A, Melo GA, Negri Filho AD, Medina MG. Regionalização e Redes de Saúde. Ciênc Saúde Colet 2018; 23:1791-8.

24. Brasil. Emenda Constitucional no 95, de 15 de dezembro de 2016. Altera o Ato das Disposições Constitucionais Transitórias, para instituir o Novo Regime Fiscal, e dá outras providências. Diário Oficial da União 2016; 15 dez.
25. Ministério da Saúde. Portaria no 2.979, de 12 de novembro de 2019. Institui o Programa Previne Brasil, que estabelece novo modelo de financiamento de custeio da Atenção Primária à Saúde no âmbito do Sistema Único de Saúde, por meio da alteração da Portaria de Consolidação no 6/GM/MS, de 28 de setembro de 2017. Diário Oficial da União 2019; 13 nov.

Recebido em 30/Abr/2020

Versão final reapresentada em 11/Mai/2020

Aprovado em 15/Mai/2020 